\title{
A case report on stage IIB testicular seminoma with hydropnephrosis and hydro ureter
}

\author{
Raviraj $S^{I}$, Thileebphan $B^{I}$
}

${ }^{1}$ Teaching Hospital, Jaffna

\section{Introduction}

Testicular Germ Cell cancers are classified into seminiferous Germ cell Tumors (SGCTs) and Non Seminomatous Germ cell Tumors (NSGCTs). The differentiation of above two entities is an important factor in Therapeutic Strategy and prognostic Index. The retroperitoneal lymphnode (RPLN) is a common site of metastasis in advanced testicular tumors, and large metastases in RPLN lead to ureteral obstruction and resultant hydronephrosis. But it can occur in early stage as happened with our patient.

(Key words :Testicular Germ cell Tumors (TGCTs),Hydronephrosis, Orchidectomy \&Chemotherapy)

\section{Case presentation}

47 years Old man with no co - morbidities presented with Left sided painless progressively increasing scrotal lump of one year. He didn't have undescended testis or previous radiation prior to this complaint. The examination revealed left side solid lump confined to scrotum. Testis couldn't separate from the lump and size was around $20 \mathrm{~cm}$. Bilateral inguinal orifices, abdomen and superficial LNs were normal.

The basic hematological including renal function and urine full reports were normal. Ultra sonography of abdomen and external genitalia revealed grossly enlarged left testis with multiple focal solid lesion favoring neoplasm. The right testis, abdomen and pelvis were normal but Beta Human Chorionic Gonadotrophin(HCG) and Lactate Dehydrogenase (LDH) levels found to be elevated.

Contrast enhanced computed tomography(CECT) of abdomen, pelvis, thorax and neck revealed multiple left para-aortic lymoh nodes(LNs) (largest $-3 \mathrm{~cm}$ ) and left iliac LNs (largest- $6 \mathrm{~cm}$ ) nodal metastasis with Left mild hydro-nephrosis and hydro ureter due to distal ureteric compression by
LNs. After multi-disciplinary meeting with consent of patient the radical orchidectomy(high) was performed and post op was uneventful.

The biopsy report revealed appearance compatible with a pure seminomatous lesion of the testis measuring about $19 \times 13 \times 7 \mathrm{~cm}$. Residual testicular tissues were not identified. There was no lymphatic invasion and no tumor extravasation. Adjuvant chemotherapy was given due to the large size of the tumor and rete testis involvement. Follow up CECT done after 4 months of orchidectomy revealed normal para-aortic LNs and improved hydronephrosis \& hydroureter.

\section{Discussion}

Testicular Germ cells tumors (TGCTs) represent $1-2 \%$ of all cancers in men. (1) But it is not a very common entity. Incidence rate of seminomatous tumors in Sri Lanka is not studied yet. The incidence is 3.79 cases $/ 100000$ men in Australia and 0.7\% in India. (1)

Peak age of Incidence is $15-35$ years. $(2,3)$ In our case the patient was 47 years and did not have distant metastatic diseases. RPLN is a common site of metastasis in advanced testicular tumors, and large metastases in RPLN occasionally lead to ureteral obstruction and resultant hydronephrosis. This complication may cause renal impairment and urinary tract infection during chemotherapy. Importantly, renal impairment limits delivery of adequate doses of anticancer drugs which might result in a lower cure rate. Therefore, effective interventions (retro grade ureteral stenting) to overcome ureteral obstruction are recommended in the management of advanced testicular tumor with severe ureteral obstruction. But our patient didn't need that as he had mild hydronephrosis and hydroureter with normal renal function tests. 
The previous TGCTs, undescended testis, Klinefelter syndrome and previous radiation to testis are the risk factors of testicular malignancy. This patient didn't have any risk factors.

Most of the patients with testicular tumors are diagnosed clinically, ultrasonically and with tumor markers as in our patient. Majority with NSGCTs have an elevated serum tumor markers such as betaHCG, LDH, Alpha Foeto Protein (AFP).But pure seminomatous tumors don't induce AFP production.

Few cases with pure seminoma according to the histopathology have been reported with elevated serum AFP levels. $(1,3)$ Anyhow if the pure seminomatous tumor patient with elevated AFP levels should be treated as cases of NSGCTs according to the guidelines. $(3,4)$ FNAC or true cut biopsy of testicular tumors is not advised due to tumor breech out along the tract. The CE CT of pelvis, abdomen, chest and neck is a guideline to detect the lymphnode and other metastatic lesion in testicular malignancy.

The staging of the tumor in this patient is stage ii b. Staging of testicular malignancy according to the Royal Marsden Hospital staging of testis cancer is given as below: Stage I-Tumor confined to testis only Stage II A-Tumor and lymph nodes involved below the diaphragm are less than $2 \mathrm{~cm}$ Stage II B-Tumor and lymph nodes involved below the diaphragm are more than $2 \mathrm{~cm}$ Stage III-Tumor and lymph nodes involved above the diaphragm and Stage IV- Tumor with distal metastasis. (5)

\section{Treatment}

Most of seminomas present with clinical stage I because of fairly large tumor. The overall survival rate of nearly $100 \%$ can be achieved by three way of management option as recommended in international guidelines. (4) First option is curative chemotherapy or radiotherapy to be implemented only in case of relapse in clinical stage I. The second option is adjuvant treatment with one course of carboplatin in all cases of stage I. The third option is considering with the prognostic factors and if the patient had bad prognostic factors they need chemotherapy. The tumor size more than $4 \mathrm{~cm}$ and invasion into rete testis and Lymphatic invasion are the recognized bad prognostic factors.
(3) Our patient is considered in this category Stage IIB need chemotherapy with or without radiation as recommended by international guidelines.

\section{Conclusion}

We report a case of pure testicular seminoma with elevated beta HCG and clinical stage IIb but with huge size and Rete testis involvemen and features of ureteral obstruction. The orchidectomy and adjuvant chemotherapy were successful.

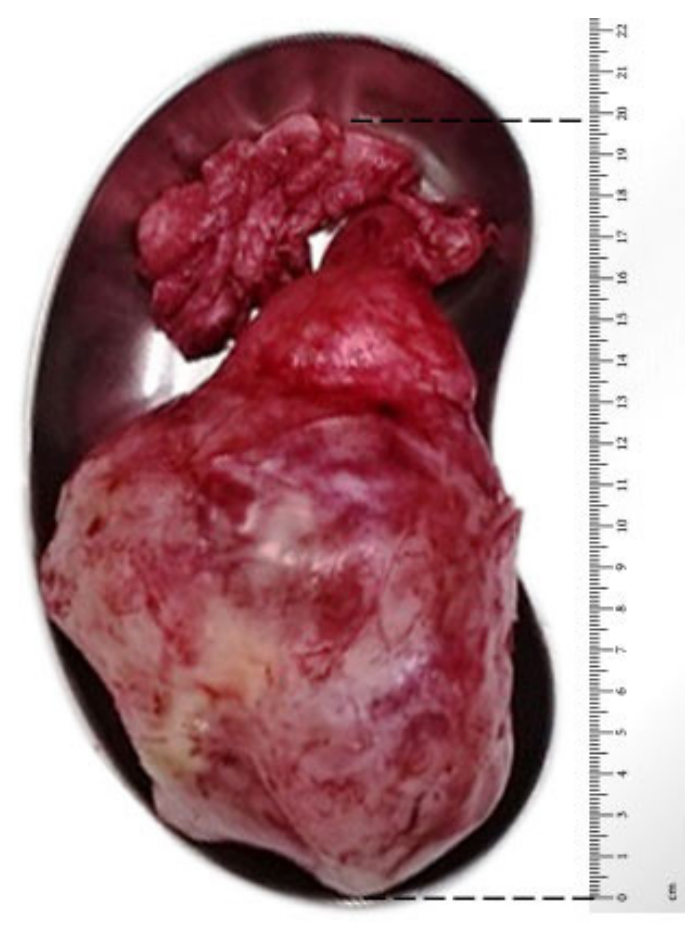

Picture 01: Depicting the orchidectomy specimen containing testis with its tumor.

\section{Learning point}

Ureteric compression and obstructive uropathy can occur in stage iib which usually reported in late advance disease. Chemotherapy may improve the condition.

\section{References}

1. Yathiraj $\mathrm{PH}$, SharanKrishna,Fernandes Donald Vidyasagar,M. Adjuvant treatment for Stage I seminoma: Why radiotherapy is better than carboplatin. Journal of Cancer Research and Therapeutics.2016:12(4): 1216-1219. Doi: 10.4103/0973-1482. 176171

2. Panza S, Santoro M, De Amicis F, Morelli C, Passarelli V, D’Aquila P, Giordano F, Cione 
E, Passarino G, Bellizzi D, et al. Estradiol via estrogen receptor beta influences ROS levels through the transcriptional regulation of SIRT3 in human seminoma TCam-2 cells. Tumour Biol. 2017 May; 39(5):1010428317701642. Doi: 10.1177/1010428317701642.

3. Di Gregorio M, Nollevaux MC, Lorge F, D'Hondt L. Metachronous testicular seminoma after radiotherapy and chemotherapy: a case report. World J Surg
Oncol. 2016 May 16;14:147. Doi: 10.1186/ s12957-016-0902-9.

4. Dieckmann, K. P. et al. Testicular seminoma clinical stage 1: treatment outcome on a routine care level. J. Cancer Res. Clin. Oncol. 142, 1599-1607 (2016).

5. Horwich A. Testicular cancer. In: Horwich A, ed. Oncology - a multidisciplinary textbook. London: Chapman and Hall, 1995485-498. 